Supporting Information

\title{
A Modular Strategy for Functional Pressure Sensitive Adhesives
}

Kyueui Lee ${ }^{1}$, Brylee David B. Tiu ${ }^{1}$, Valentin Martchenko ${ }^{2}$, Kristene Mai ${ }^{2}$, Goun Lee ${ }^{3}$, Matthias Gerst $^{4}$, Phillip B. Messersmith ${ }^{*}, 1,5,6$

${ }^{1}$ Department of Bioengineering, University of California at Berkeley, Berkeley, CA 94720, USA

${ }^{2}$ Department of Chemical Biology, University of California at Berkeley, Berkeley, CA 94720, USA

${ }^{3}$ Department of Molecular and Cellular Biology, University of California at Berkeley, Berkeley, CA 94720, USA

${ }^{4}$ Polymers for Adhesives, BASF SE, D-67056 Ludwigshafen, Germany

${ }^{5}$ Department of Materials Science and Engineering, University of California at Berkeley, Berkeley, CA 94720, USA

${ }^{6}$ Materials Sciences Division, Lawrence Berkeley National Laboratory, Berkeley, CA 94720, USA

*Corresponding Author - Phillip B. Messersmith (E-mail: philm@ berkeley.edu) 


\section{TABLE OF CONTENTS}

1. General Information $\quad 3$

2. Synthesis and Characterization of Scaffold Pressure Sensitive Adhesive 3

2.1. Synthetic Procedures 3

2.2. Monomer Conversion Ratio 3

2.3. Actual Polymer Composition 4

3. Synthesis and Characterization of Aliphatic and Benzoic PSAs 6

3.1. Synthetic Procedures $\quad 6$

3.2. Polymer Conversion Ratio $\quad 7$

4. Synthesis and Characterization of Bioinspired PSAs 8

4.1. N-(3,4-bis((tert-butyldimethylsilyl)oxy)phenethyl)-4-mercaptobutanamide Synthesis 8

4.2. 4-mercapto-N-phenethylbutanamide Synthesis 10

4.3. Polymer Synthesis 12

4.4. Polymer Conversion Ratio 13

4.5. Molecular Weight and Polydispersity 14

4.6. Deprotection of Protecting Groups 15

4.7. Glass Transition Temperature 17

5. Mechanical Tests 17

6. Time-dependent Adhesion/Cohesion Behaviors in Catecholic PSAs 18

6.1. Mechanical Tests 18

6.2. UV-vis Spectroscopy 18

7. Statistical Analysis $\quad 19$

8. References 19 


\section{General Information}

Most of chemicals were purchased from Sigma-Aldrich (Saint Louis, MO, US) unless otherwise specified. The ${ }^{1} \mathrm{H}(400 \mathrm{MHz})$ and ${ }^{13} \mathrm{C}$ NMR $(100 \mathrm{MHz})$ spectra were collected by using Bruker AVB-400 spectrometer (Billerica, MA, US).

\section{Synthesis and Characterization of Scaffold Pressure Sensitive Adhesive}

\subsection{Synthetic Procedures}

The scaffold pressure sensitive adhesive (PSA), P1, was synthesized by free radical polymerization in anhydrous dimethylformamide (DMF; Acros Organics, Geel, Belgium, 99.8\%) using butyl acrylate (BA; $\geq 99 \%$ ), tert-butyl acrylate (TBA; 98\%), and glycidyl methacrylate (GMA; $\geq 97.0 \%$ ). Prior to the polymerization, all the acrylates were passed through a column packed with inhibitor removers, and a radical initiator azobisisobutyronitrile (AIBN, $\geq 98 \%$ ) was recrystallized in methanol. Then, $250 \mathrm{~mL}$ of anhydrous DMF, AIBN (1.2 g, $7.3 \mathrm{mmol})$, BA (80.5 $\mathrm{g}, 627.8 \mathrm{mmol})$, TBA $(7.9 \mathrm{~g}, 61.4 \mathrm{mmol})$, and GMA $(6.5 \mathrm{~g}, 45.4 \mathrm{mmol})$ were mixed in a $500 \mathrm{~mL}$ Schlenk flask using a Teflon stirring bar. The solution mixture was degassed by freeze-pump-thaw cycles (repeated three times) and the flask was filled with Ar gas (Praxair, Danbury, CT, US, $\geq 99.999 \%$ ). The polymerization was initiated by immersing the flask in an oil bath at $60{ }^{\circ} \mathrm{C}$ and the reaction was carried out overnight. To quench the reaction, the solution mixture was frozen by liquid $\mathrm{N}_{2}$ and subsequently thawed in a water bath. The polymer was then precipitated in methanol (Fisher Scientific, Waltham, MA, US, 99\%)/water 95:5 (\%, v/v) co-solvent to remove unreacted monomers and others. Approximately $50 \mathrm{~mL}$ of tetrahydrofuran (THF; Fisher Scientific, Waltham, MA, US, $\geq 99.9 \%$ ) was used to re-dissolve the polymer, and the precipitation cycle was repeated two or more times. After the final precipitation, the polymer was dried under high vacuum to remove solvents.

\subsection{Monomer Conversion Ratio}

To determine the monomer conversion ratio, a chemical mixture collected right after the polymerization reaction was analyzed by ${ }^{1} \mathrm{H}$ NMR spectroscopy (Figure S1). The conversion ratio was calculated by the following equation:

$$
\text { conversion ratio }(\%)=\frac{(H b / 2)}{(H b / 2)+(H a / 1)} \times 100
$$

where $H a$ is the integral area of the vinyl peak in the unreacted BA monomer $(1 \mathrm{H}, 6.3 \mathrm{ppm})$, and $\mathrm{Hb}$ is the integral area of the first methylene peak in the BA side chain from the polymer backbone (2H, $3.9 \mathrm{ppm})$. 


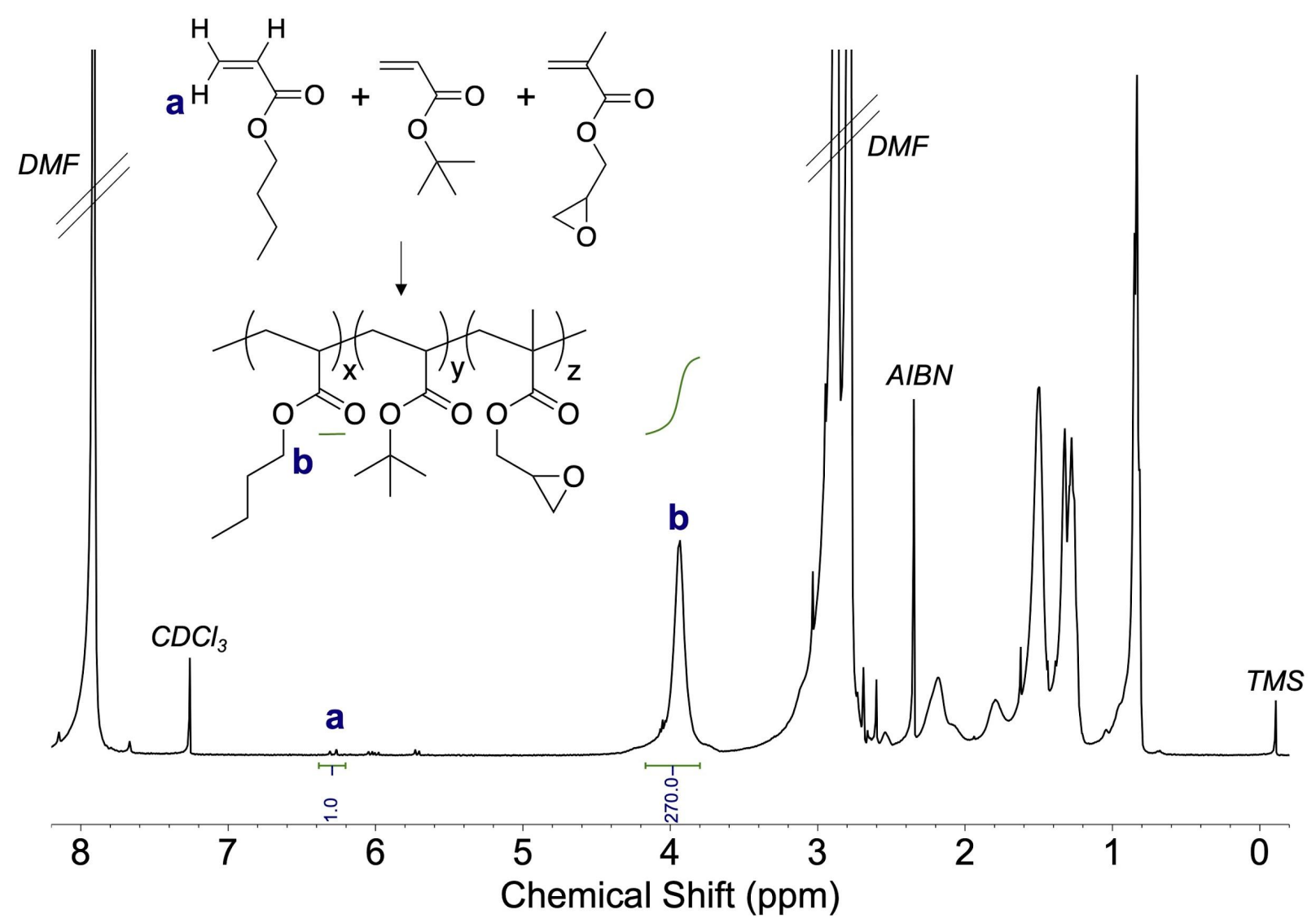

Figure S1. ${ }^{1} \mathrm{H}$ NMR spectrum of a chemical mixture after polymerization.

\subsection{Actual Polymer Composition}

The actual molar ratio (\%) between BA, TBA and GMA side chains in the scaffold PSA was determined by ${ }^{1} \mathrm{H}$ NMR spectroscopy (Figure S2) by the following equations:

$$
\begin{gathered}
H x(b)+H y(d)=15.59 \mathrm{~Hz} \\
3 H x(j)+3 H z(f)=44.52 \mathrm{~Hz}
\end{gathered}
$$

where $H x, H y$ and $H z$ are standardized integral areas (1H) of BA, TBA and GMA side chains, respectively. Alphabetic indications in the round brackets represent corresponding NMR peaks assigned in Figure S2. From the two equations above, the ratio between $H x, H y$, and $H z$ can be derived as follows:

$$
H x: H y: H z=13.31: 1.75: 1=83: 11: 6
$$




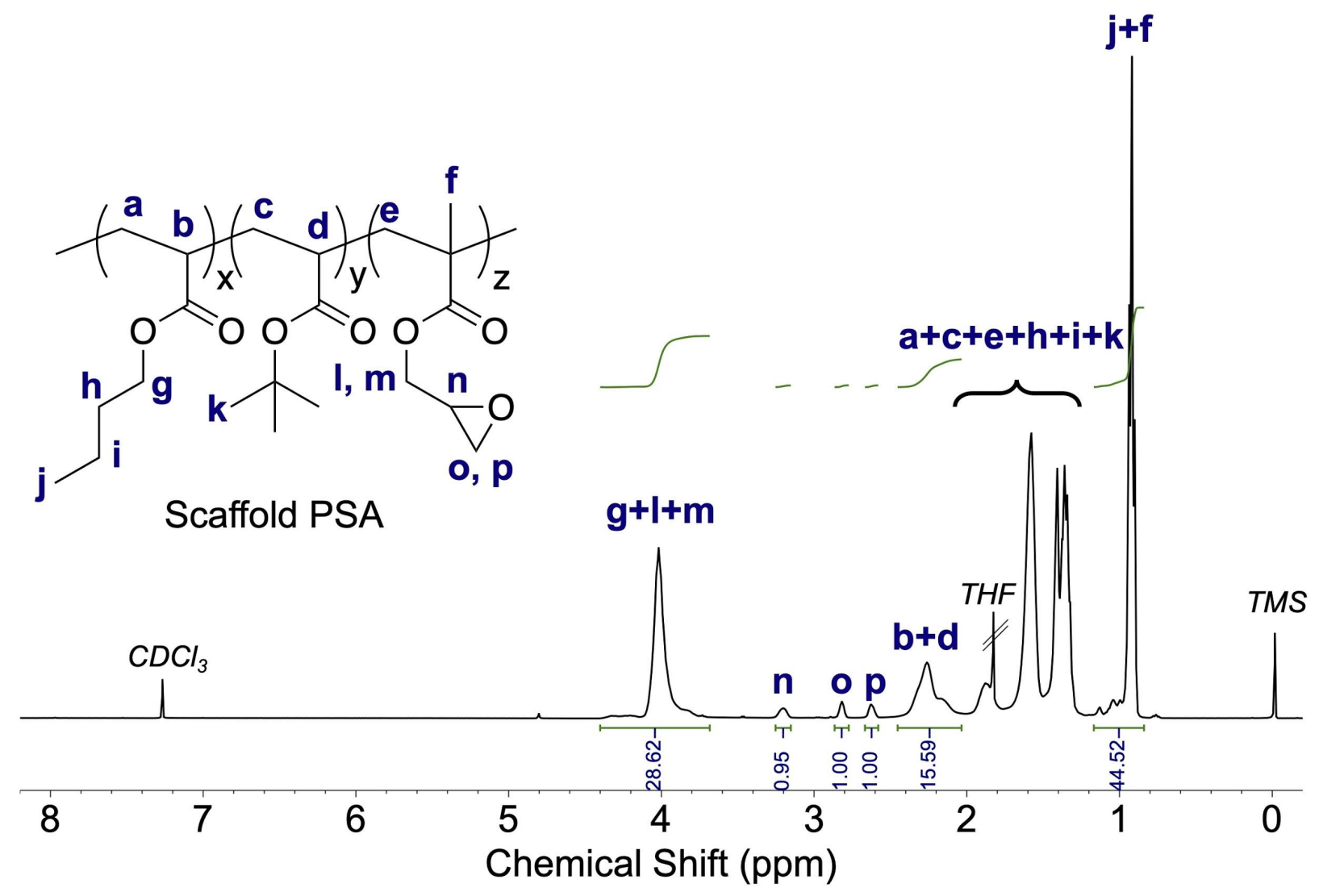

Figure S2. ${ }^{1} \mathrm{H}$ NMR spectrum of the scaffold PSA. 


\section{Synthesis and Characterization of Aliphatic and Benzoic PSAs}

\subsection{Synthetic Procedures}

\section{Scaffold PSA}

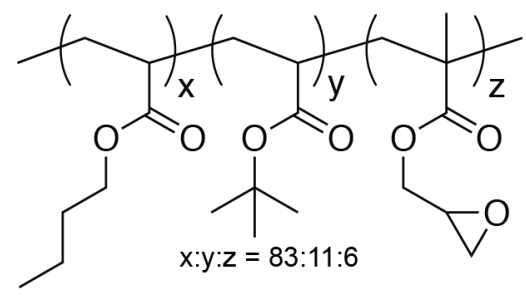

P1

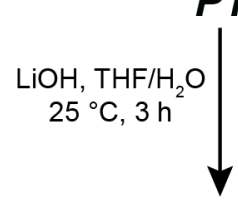<smiles>[Z]C(C)(C)C(C)(C)C(=O)OCC(O)CSCCO</smiles>

Aliphatic Thiol

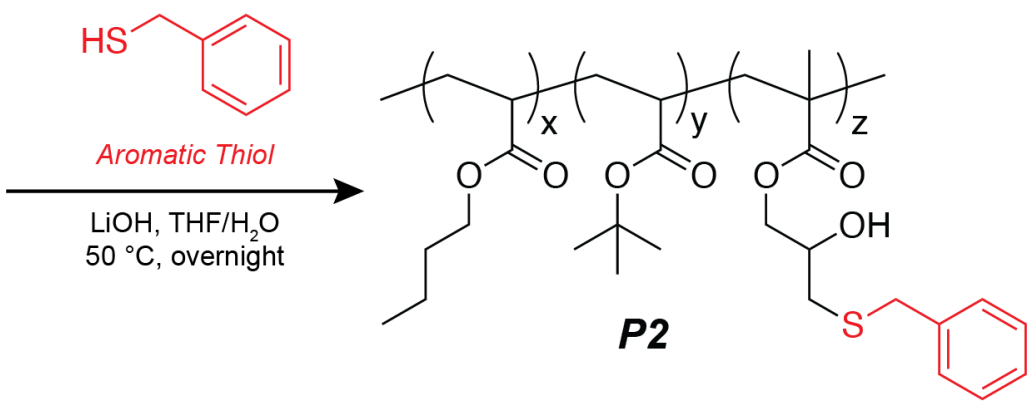

Figure S3. Chemical synthesis of post-modified PSAs using aromatic and aliphatic thiols.

Aliphatic PSA, P2: The scaffold polymer, P1, $(1.0 \mathrm{~g}, 0.465 \mathrm{mmol}$ of the epoxide side chain in the polymer backbone, 1 eq.) and 2-mercapto-1-ethanol ( $\geq 99.0 \%, 43.5 \mu \mathrm{L}, 0.618 \mathrm{mmol}, 1.33$ eq.) were dissolved in Ar-bubbled anhydrous THF ( $\geq 99.9 \%$ ) ( $8 \mathrm{~mL}$ and $1 \mathrm{~mL}$, respectively). The solutions were combined in a round bottom flask and subsequently sealed with rubber septa. After 10 minutes of Ar-bubbling, $\mathrm{LiOH}(0.028 \mathrm{~g}, 0.117 \mathrm{mmol}, 0.25 \mathrm{eq}$.) dissolved in deionized water (1.0 $\mathrm{mL})$ was added to the mixture using a syringe and allowed to react in an oil bath $\left(50{ }^{\circ} \mathrm{C}\right)$ with constant stirring for 3 hours. After the reaction, the mixture solution was cooled to room temperature and further concentrated using a rotary evaporator until a viscous mixture was obtained. Then, the mixture was precipitated in Ar-bubbled cold hexane $(\geq 98.5 \%, 200 \mathrm{~mL})$. The precipitate was re-dissolved in HPLC grade THF ( $\geq 99.9 \%, 10 \mathrm{~mL})$ and underwent two additional precipitation cycles. The final precipitate was dried under a high vacuum.

Benzoic PSA, P3: The experimental procedures are identical to the above description except for the molar equivalent of a grafting reagent, benzyl mercaptan ( $\geq 99 \%, 2$ eq.), and reaction time (overnight). 


\subsection{Polymer Conversion Ratio}

Conversion ratios of the scaffold polymer for the post-modified aliphatic and benzoic PSAs were calculated by ${ }^{1} \mathrm{H}$ NMR spectroscopy (Figure S3) by the following equations:

$$
\text { conversion ratio }(\%)=\frac{(H r / 2)}{(H r / 2)+(H n / 1)} \times 100
$$

where $H n$ is the integral area of the epoxide peak from the scaffold polymer $(1 \mathrm{H}, 3.2 \mathrm{ppm})$, and $\mathrm{Hr}$ is the integral area of the methylene peak adjacent to sulfide from the post-modified polymers (2H, $2.9 \mathrm{ppm}$ and $3.8 \mathrm{ppm}$ for aliphatic and benzoic polymers, respectively).

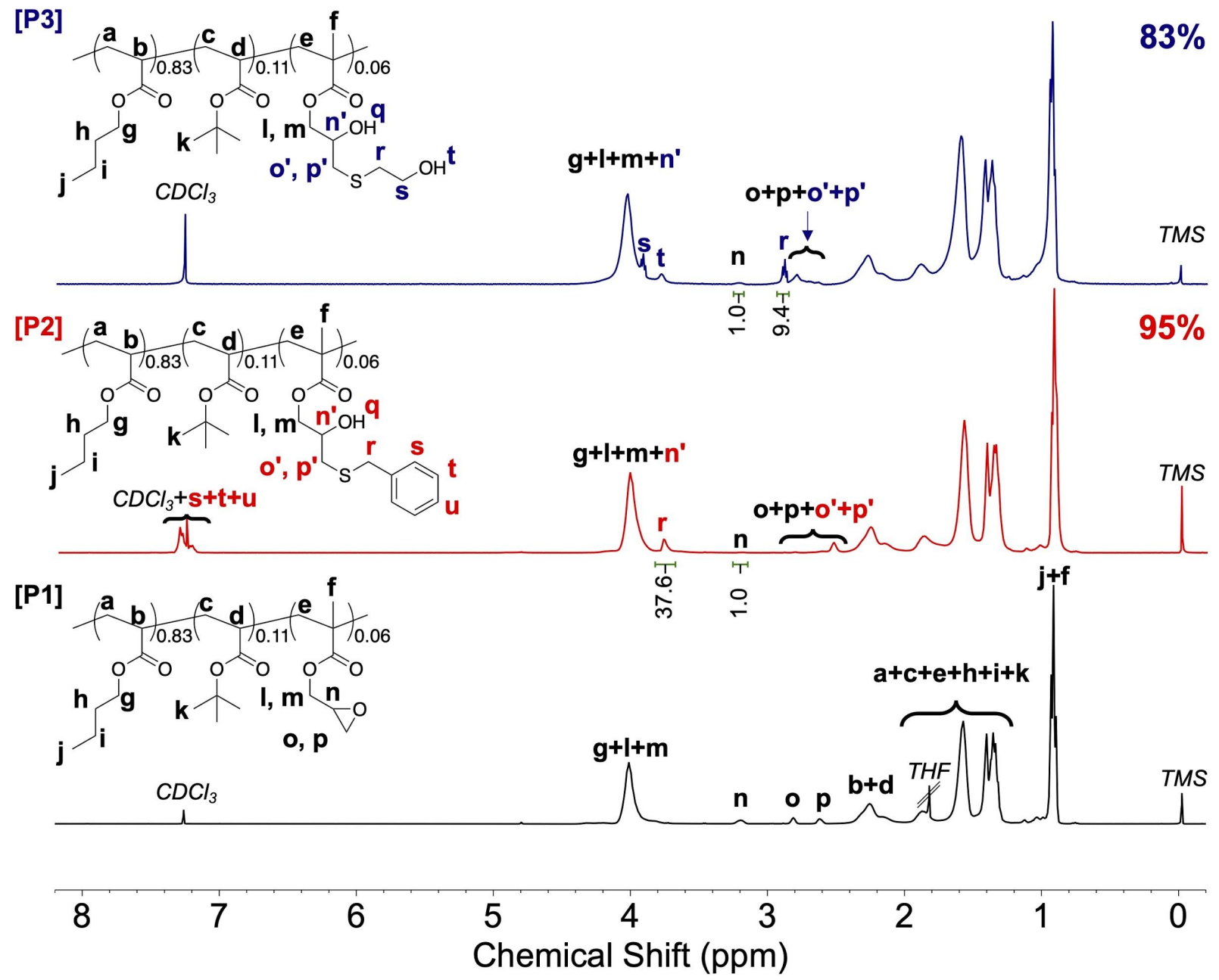

Figure S4. ${ }^{1} \mathrm{H}$ NMR spectrum of the scaffold (P1), benzoic (P2) and aliphatic (P3) PSAs. 


\section{Synthesis and Characterization of Bioinspired PSAs}

\subsection{N-(3,4-bis((tert-butyldimethylsilyl)oxy)phenethyl)-4-mercaptobutanamide Synthesis}

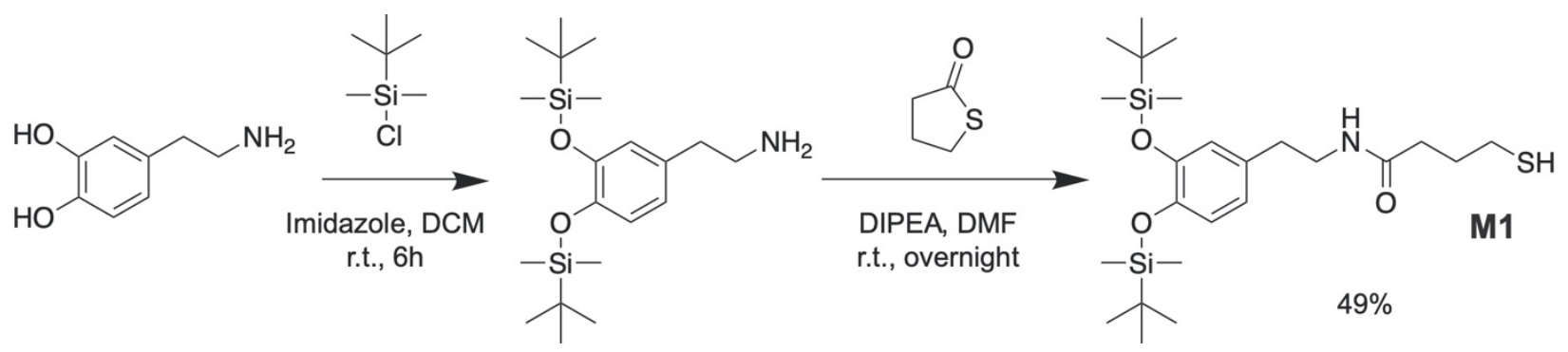

Figure S5. A schematic of N-(3,4-bis((tert-butyldimethylsilyl)oxy)phenethyl)-4mercaptobutanamide (M1) synthesis

M1 was synthesized by the following procedures. Step 1: In a $250 \mathrm{~mL}$ round bottom flask, dopamine hydrochloride (Alfa Aesar, Haverhill, MA, US, 99\%, 9.999 g, 53 mmol, 1 eq.) and imidazole (11.67 g, $171 \mathrm{mmol}, 3.25$ eq.) were dissolved in $50 \mathrm{~mL}$ Ar-bubbled anhydrous dichloromethane (DCM; 99.8\%) with magnetic stirring. In a separate round bottom flask, tertbutyldimethylsilyl chloride (97\%, $19.866 \mathrm{~g}, 135 \mathrm{mmol}, 2.5 \mathrm{eq}$.) was dissolved in Ar-bubbled anhydrous DCM $(55 \mathrm{~mL})$ and added dropwise to the dopamine hydrochloride solution. After 10 minutes of Ar gas exchange, the mixture reacted under an Ar balloon at room temperature for $6 \mathrm{~h}$ with stirring. Liquid-liquid extraction was performed by diluting the reaction mixture in DCM (Fisher Scientific, Waltham, MA, US, 99.9\%, $300 \mathrm{~mL}$ ) and washing 5 times with deionized water $(150 \mathrm{~mL})$ and 5 times saturated aqueous $\mathrm{NH}_{4} \mathrm{Cl}$ solution $(150 \mathrm{~mL})$. The final organic layer was stirred with $75 \mathrm{~g}$ of anhydrous $\mathrm{MgSO}_{4}$ and subjected to vacuum filtration. The filtrate was thoroughly condensed using rotary evaporation and Schlenk line to yield a white powdery solid with a brown tint. Step2: In a $250 \mathrm{~mL}$ round bottom flask with a Teflon stir bar, TBS-protected dopamine (17.254 g, 45 mmol, 1 eq.) was dissolved in Ar-bubbled anhydrous DMF (90.4 mL). The flask was sealed and allowed to Ar-exchange. N, N-diisopropylethylamine (99.5\%, $23.62 \mathrm{~mL}$, $136 \mathrm{mmol}, 3$ eq.) followed by $\gamma$-thiobutyrolactone $(98 \%, 5.20 \mathrm{~mL}, 60 \mathrm{mmol}, 1.33$ eq.) were added dropwise to sealed reaction mixture. The reaction ran under an Ar balloon overnight at room temperature with stirring. The solution was then diluted with DCM $(200 \mathrm{~mL})$ and washed with deionized water $(200 \mathrm{~mL})$ followed by brine $(150 \mathrm{~mL})$ for 5 times each. The final organic layer was dried with $\sim 75 \mathrm{~g}$ of magnesium sulfate (Oakwood Chemical, Hampton County, SC, US), subjected to vacuum filtration, and further condensed with silica gel $(\sim 30 \mathrm{~g})$ through rotary evaporation. A pre-absorbed sample of silica gel was added evenly on the top of a column, where bare silica gel (AC360062500, Acros Organics, Geel, Belgium) was saturated with mobile phase co-solvent (2:1 hexane/ethyl acetate (99.5\%)) and packed six inches of the column. Approximately $1 \mathrm{~cm}$ of sand was added to the column. Column chromatography was carried out concurrently with thin-layer chromatography (TLC) to detect M1 from collected fractions (The sample was 
confirmed by both UV-light and Ellman's reagent staining). The column chromatography fractions were combined and dried under high vacuum to produce a white solid powder (49\% yield). ${ }^{1} \mathrm{H}$ NMR (400 MHz, DMSO- $\left.d_{6}\right) \delta 7.85(\mathrm{t}, J=5.6 \mathrm{~Hz}, 1 \mathrm{H}), 6.79-6.61(\mathrm{~m}, 3 \mathrm{H}), 3.20(\mathrm{q}, J=6.8 \mathrm{~Hz}$, $2 \mathrm{H}), 2.57(\mathrm{t}, J=7.3 \mathrm{~Hz}, 2 \mathrm{H}), 2.42(\mathrm{q}, J=7.4 \mathrm{~Hz}, 2 \mathrm{H}), 2.22(\mathrm{t}, J=7.7 \mathrm{~Hz}, 1 \mathrm{H}), 2.13(\mathrm{t}, J=7.3 \mathrm{~Hz}$, $2 \mathrm{H}), 1.72(\mathrm{p}, J=7.2 \mathrm{~Hz}, 2 \mathrm{H}), 0.94(\mathrm{~d}, J=3.6 \mathrm{~Hz}, 18 \mathrm{H}), 0.16(\mathrm{~d}, J=3.8 \mathrm{~Hz}, 12 \mathrm{H}) .{ }^{13} \mathrm{C}$ NMR $(101$ MHz, DMSO- $\left.d_{6}\right) \delta 171.70,146.21,144.83,133.29,122.19,121.69,120.92,40.68,34.89,34.40$, $30.05,26.20,23.96,18.54,-3.76$.

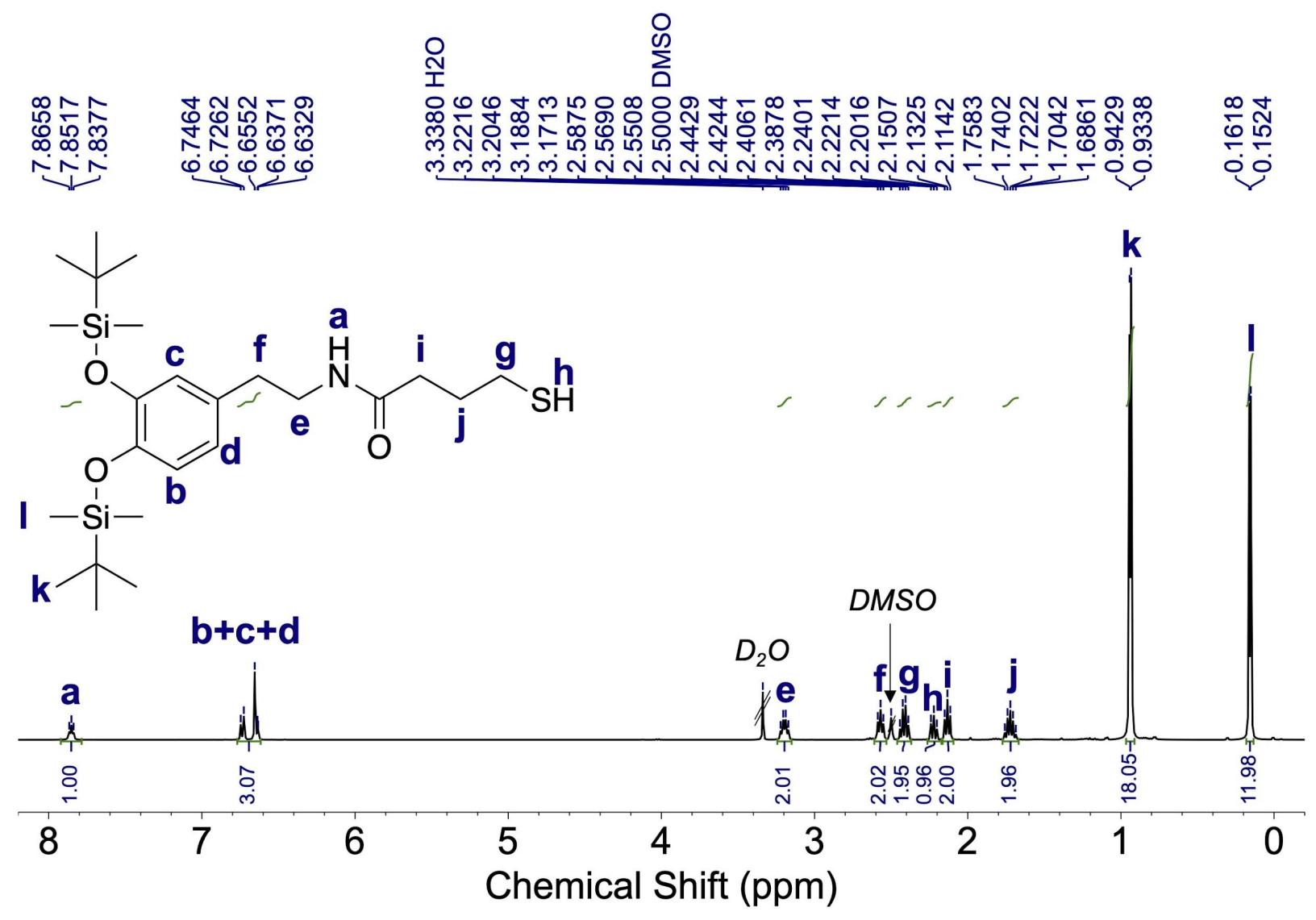

Figure S6. ${ }^{1} \mathrm{H}$ NMR spectrum of M1. 


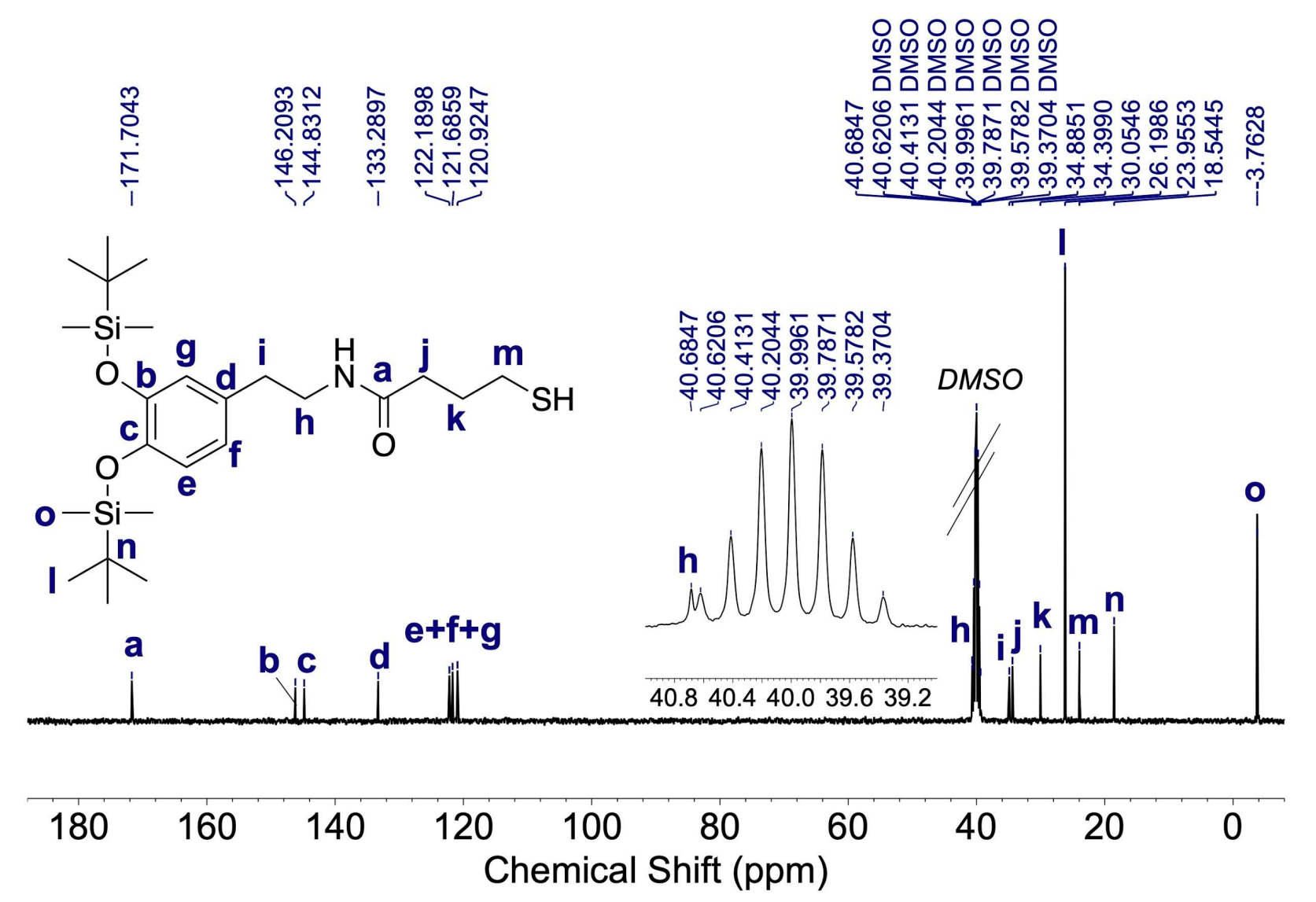

Figure S7. ${ }^{13} \mathrm{C}$ NMR spectrum of M1.

\subsection{4-mercapto-N-phenethylbutanamide Synthesis}

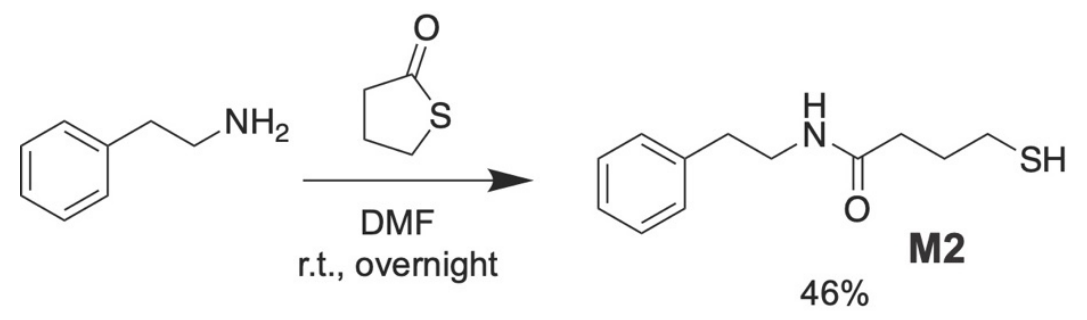

Figure S8. A schematic of 4-mercapto-N-phenethylbutanamide (M2) synthesis

In Ar-bubbled anhydrous DMF (104.79 mL), phenethylamine (98\%, $20.75 \mathrm{~mL}, 52 \mathrm{mmol}$, 1 eq.) and $\gamma$-thiobutyrolactone $(6.03 \mathrm{~mL}, 70 \mathrm{mmol}, 1.33$ eq. $)$ were added dropwise with constant stirring. The flask was subsequently sealed with rubber septa and Ar-exchange was allowed for 10 minutes. The solution mixture was kept at room temperature with constant stirring for an overnight reaction. The solution was then diluted with DCM (200 mL). Extractions followed by purification using column chromatography were performed as described above, except for the composition of mobile phase co-solvent (1:2 hexane/ethyl acetate for column chromatography. The selected fractions were combined and dried under high vacuum to produce a white solid powder $(46 \%$ 
yield). ${ }^{1} \mathrm{H}$ NMR (400 MHz, DMSO- $\left.d_{6}\right) \delta 7.91(\mathrm{t}, J=5.7 \mathrm{~Hz}, 1 \mathrm{H}), 7.44-6.95(\mathrm{~m}, 5 \mathrm{H}), 3.26(\mathrm{q}, J$ $=6.7 \mathrm{~Hz}, 2 \mathrm{H}), 2.69(\mathrm{t}, J=7.4 \mathrm{~Hz}, 2 \mathrm{H}), 2.41(\mathrm{q}, J=7.3 \mathrm{~Hz}, 2 \mathrm{H}), 2.27(\mathrm{t}, J=7.8 \mathrm{~Hz}, 1 \mathrm{H}), 2.14(\mathrm{t}$, $J=7.3 \mathrm{~Hz}, 2 \mathrm{H}), 1.72(\mathrm{p}, J=7.2 \mathrm{~Hz}, 2 \mathrm{H}) .{ }^{13} \mathrm{C}$ NMR $\left(101 \mathrm{MHz}, \mathrm{DMSO}-d_{6}\right) \delta 171.81,139.98$, $129.08,128.73,126.49,40.54,35.65,34.41,30.07,23.93$.

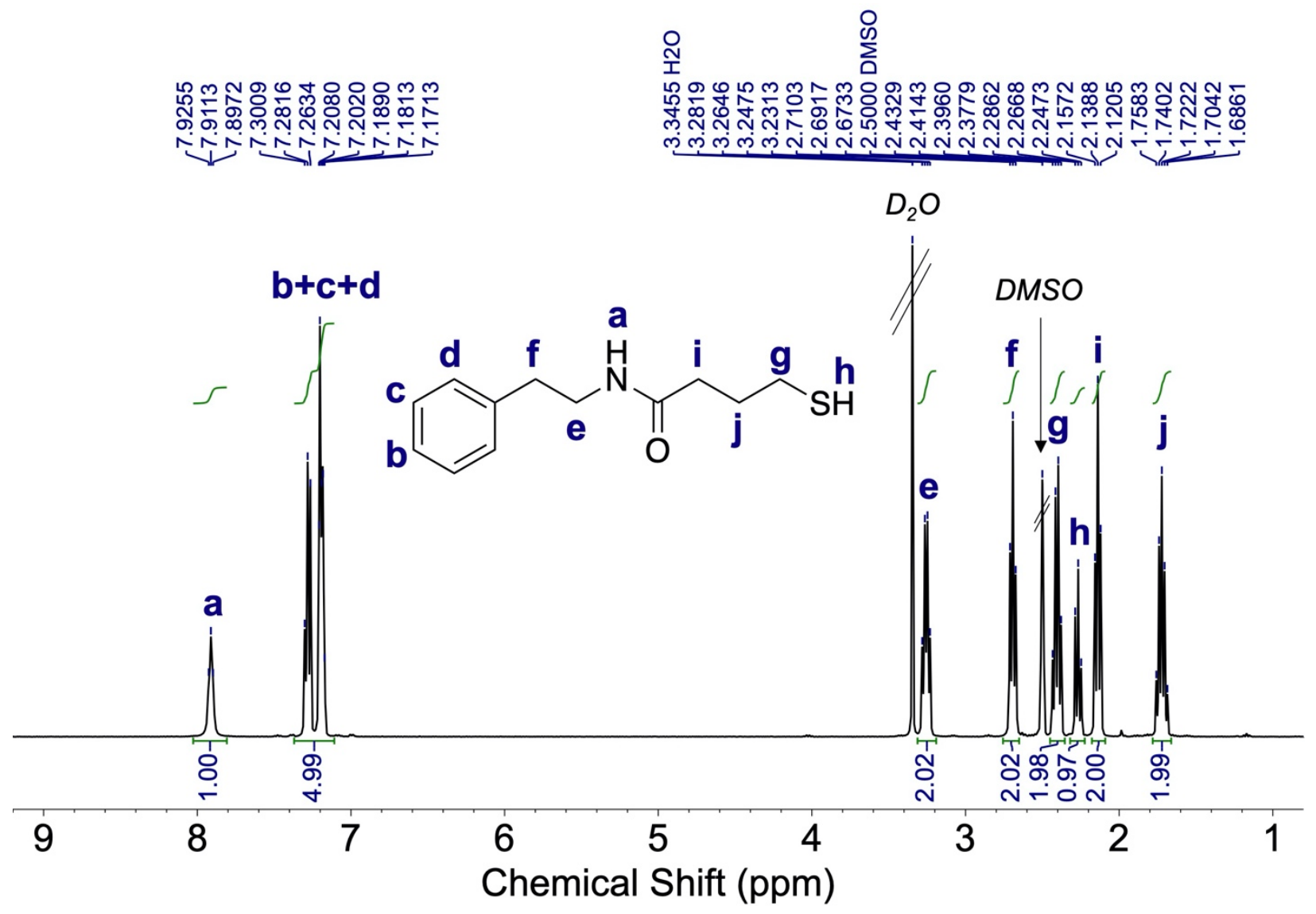

Figure S9. ${ }^{1} \mathrm{H}$ NMR spectrum of M2. 


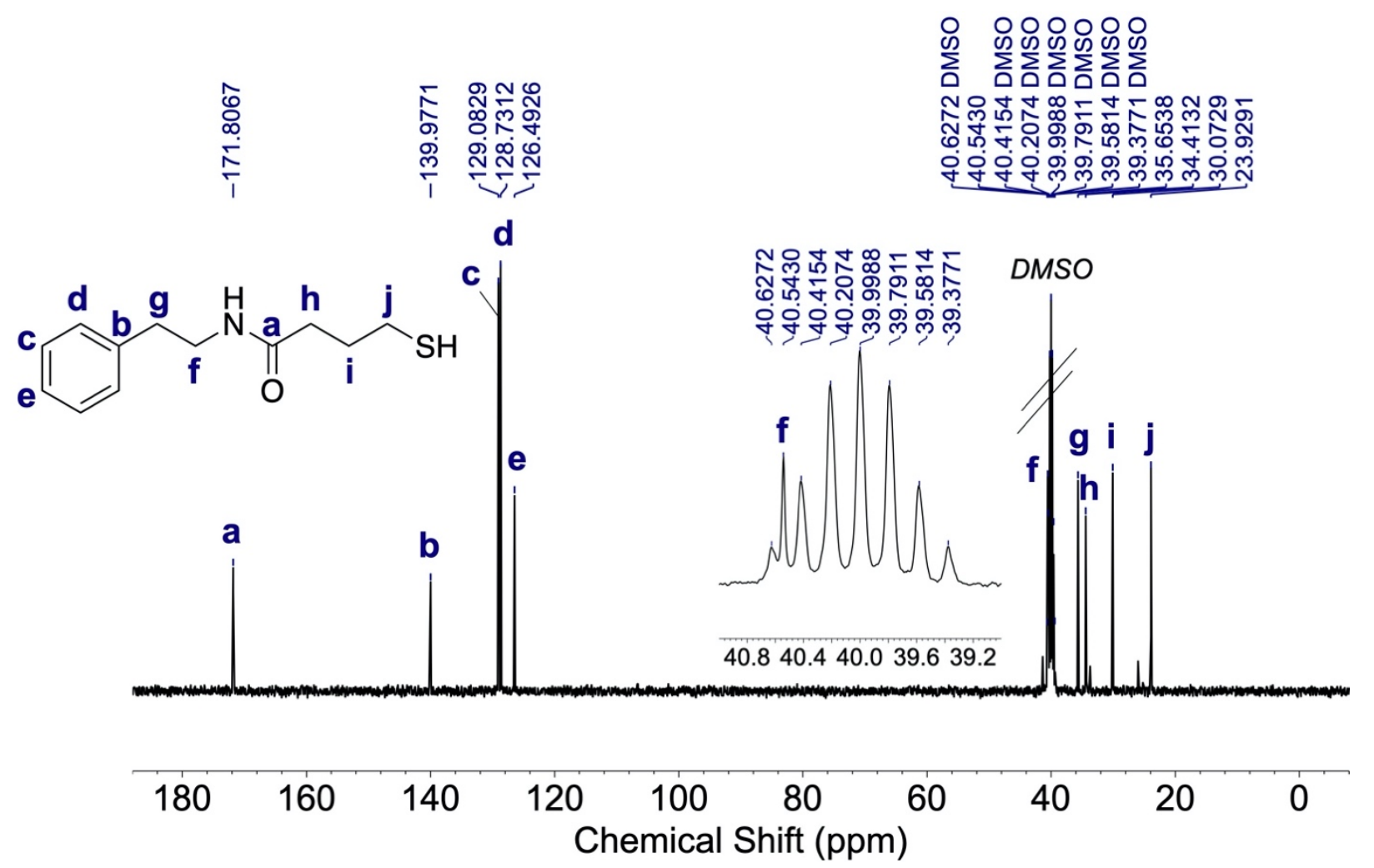

Figure S10. ${ }^{13} \mathrm{C}$ NMR spectrum of M2.

\subsection{Polymer Synthesis}

Catecholic PSA, P4: The scaffold polymer $(1.0 \mathrm{~g}, 0.465 \mathrm{mmol}$ of the epoxide side chain in the polymer backbone, 1 eq.) and $\mathrm{M} \quad(0.299 \mathrm{~g}, 0.618 \mathrm{mmol}, 1.33)$ were dissolved in Ar-bubbled anhydrous THF $(9 \mathrm{~mL}$ and $1 \mathrm{~mL}$, respectively). The two solutions were mixed with 1,8Diazabicyclo[5.4.0]undec-7-ene (DBU; 98\%, $14.4 \mu \mathrm{L}, 93 \mu \mathrm{mol}, 0.20$ eq.) in a sealed Ar gasexchanged flask with stirring and placed in an oil bath $\left(55^{\circ} \mathrm{C}\right)$ for 30 hours. The solution was dried by rotary evaporation until a clear viscous mixture remained. The polymer was purified from the mixture using the precipitation steps outlined in section 3.1.

Benzoic PSA, P6: The grafting of M2 ( $0.147 \mathrm{~g}, 0.618 \mathrm{mmol}, 1.33$ eq. $)$, to the scaffold polymer was achieved by following the above description.

Deprotection of protecting groups in the PSAs (P4 and P6), P5 and P7: TBS and tert-butyl groups in P4 and P6 were deprotected by dissolving the PSAs (10g) in Ar-bubbled anhydrous DCM (100 $\mathrm{mL})$ with trifluoroacetic acid (TFA; 99\%) $(50 \mathrm{~mL})$ and deionized water $(2.5 \mathrm{~mL})$. The flask containing the mixture solution was instantly sealed and reacted overnight. Each polymer was purified from the mixture through three precipitation cycles outlined in section 3.1. 


\subsection{Polymer Conversion Ratio}

Conversion ratios of the scaffold polymer (P1) for P4 and P6 were calculated by ${ }^{1} \mathrm{H}$ NMR spectroscopy (Figure S11) by the following equations:

$$
\text { conversion ratio }(\%)=\frac{(H v / 2)}{(H v / 2)+(H n / 1)} \times 100
$$

where $H n$ is the integral area of the epoxide peak from the scaffold polymer $(1 \mathrm{H}, 3.2 \mathrm{ppm})$, and $H v$ is the integral area of the methylene peak adjacent to secondary amine from the post-modified polymers (2H, $3.5 \mathrm{ppm}$ and $3.4 \mathrm{ppm}$ for benzoic and catecholic PSAs, respectively).

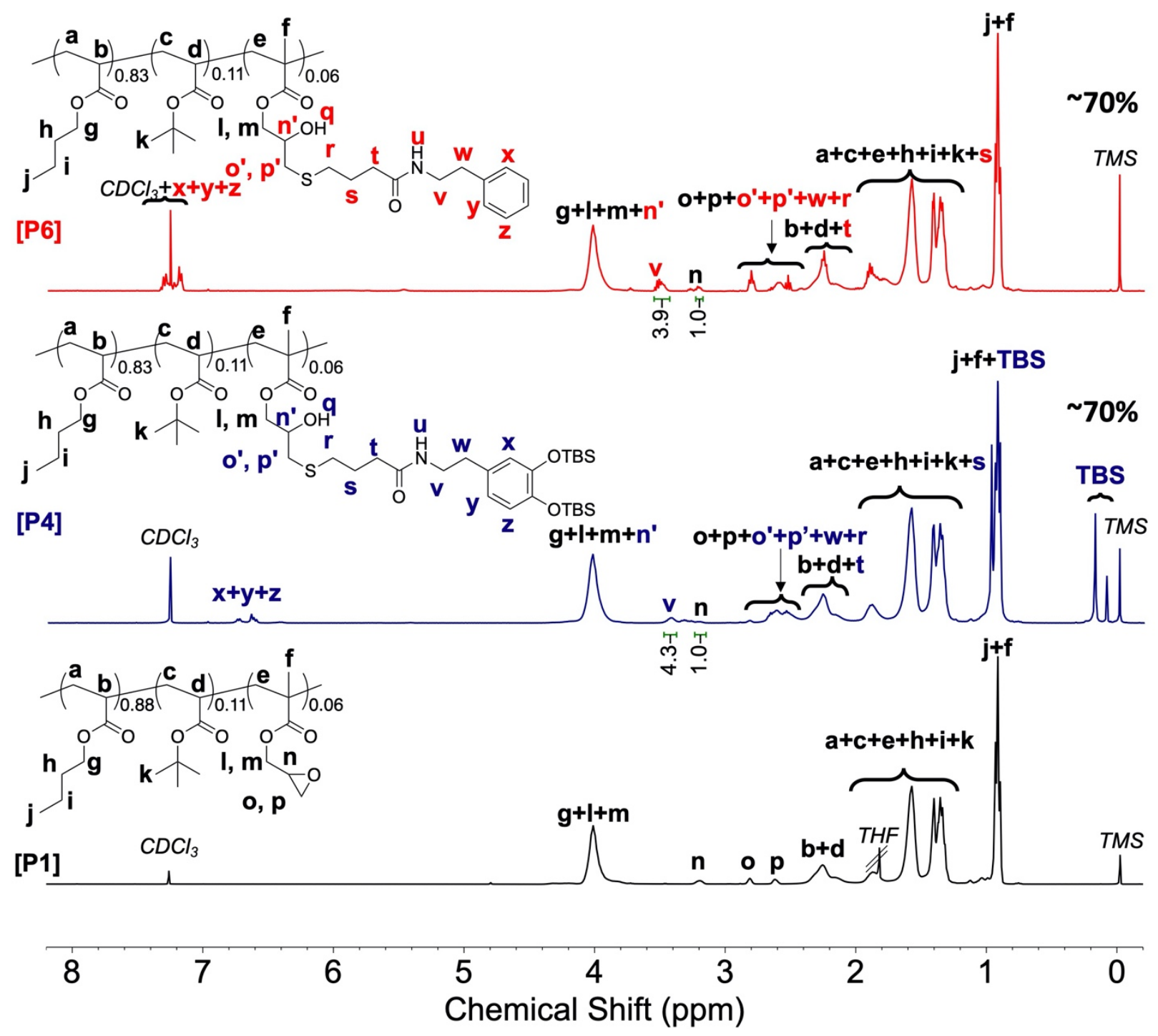

Figure S11. ${ }^{1} \mathrm{H}$ NMR spectrum of the scaffold (P1), benzoic (P4) and catecholic (P6) PSAs. 


\subsection{Molecular Weight and Polydispersity}

Molecular weights and polydispersity of PSAs were calculated by gel permeation chromatography (GPC) analysis where polystyrene calibration standards (Agilent EasiVial, Santa Clara, CA, US) were used for calibration. The polymers were eluted using THF through a TSKGEL SUPER HM-M 6X150 (Tosoh Bioscience, Tokyo, Japan) with a flow rate of $0.3 \mathrm{~mL} / \mathrm{min}$, which was connected to the Waters Alliance HPLC 2695 separation module system (Milford, MA, US) with a 2414 refractive index detector.

Additional Discussion: Molecular weight of scaffold and functionalized PSA was similar (Table 3); however, the scaffold PSA showed a slightly higher Mw value compared to those of the functionalized PSAs. This may be caused by a change in solubility due to the grafted polar functional groups or possible chemical interaction between grafted functional groups and the GPC column.

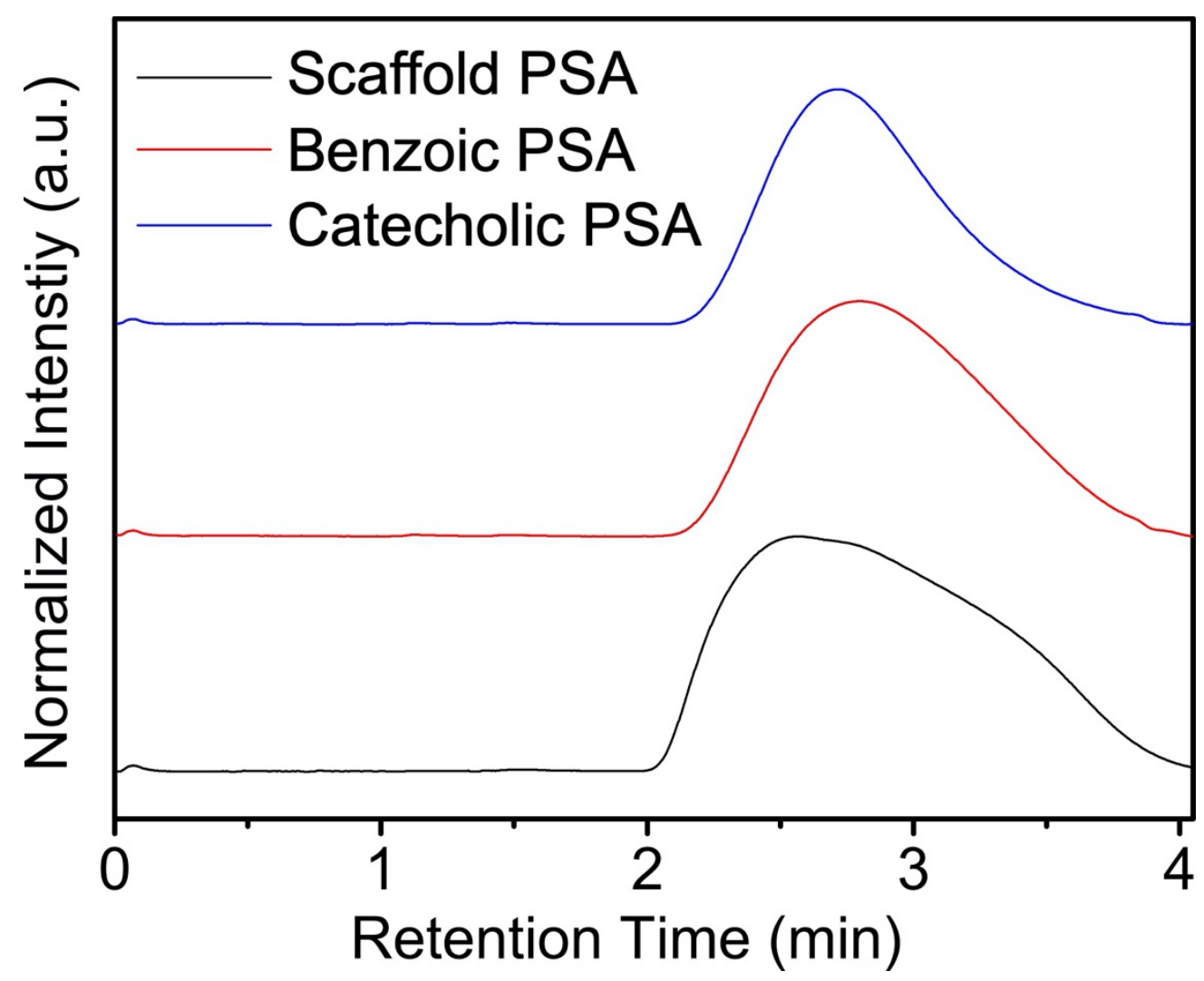

Figure S12. Normalized GPC curves from scaffold (P1), catecholic (P4) and benzoic (P6) PSAs. 


\subsection{Deprotection of Protecting Groups}

Catecholic PSA, P5:

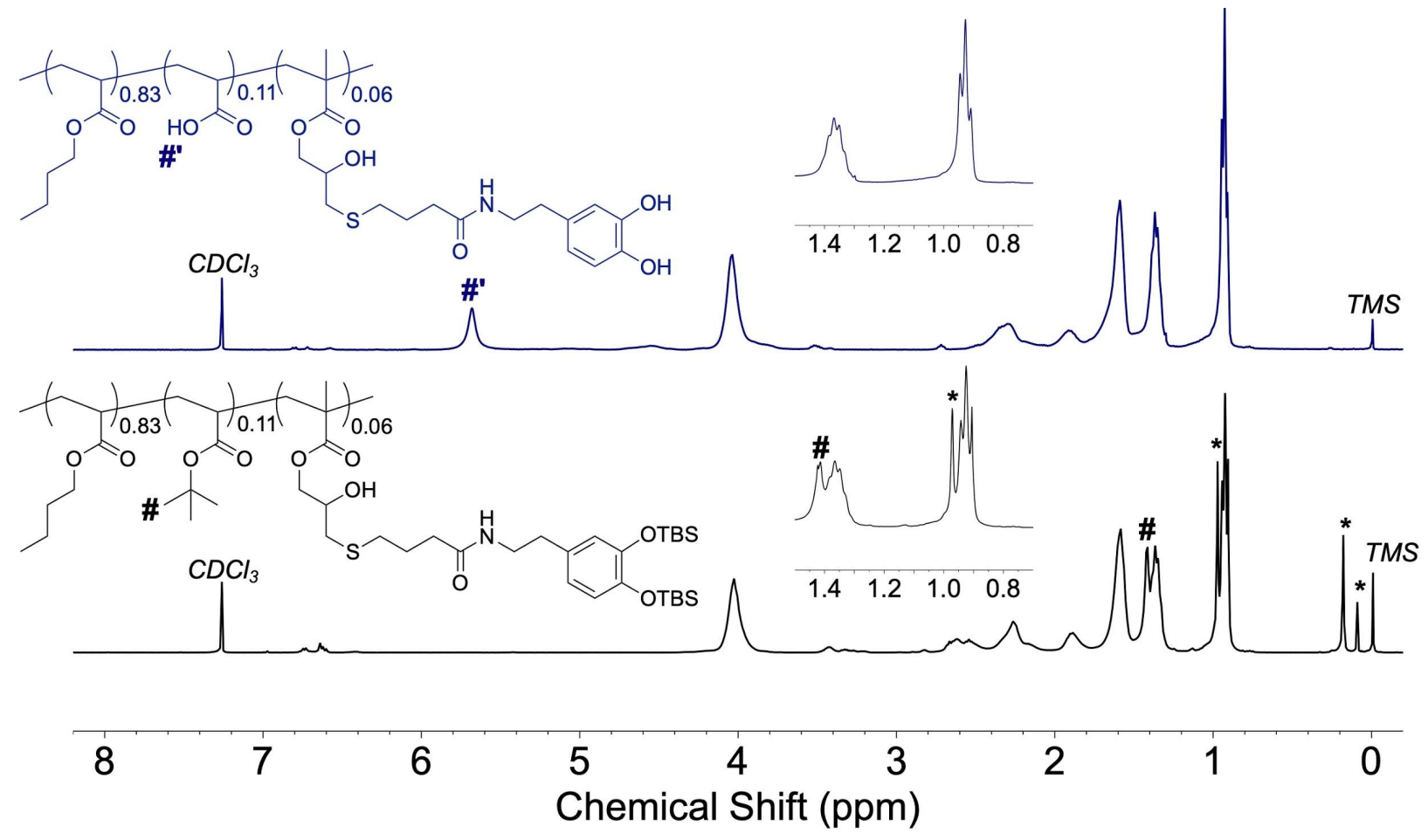

Figure S13. ${ }^{1} \mathrm{H}$ NMR spectrum of catecholic PSAs before (P4, black) and after (P5, blue) deprotection process. The pound sign (\#) indicates the peak from the tert-butyl group, which is substituted for the carboxylic group (\#') after deprotection. The asterisk indicates the peaks from TBS groups (P5), which are successfully removed by the deprotection process. 
Benzoic PSA, P7:

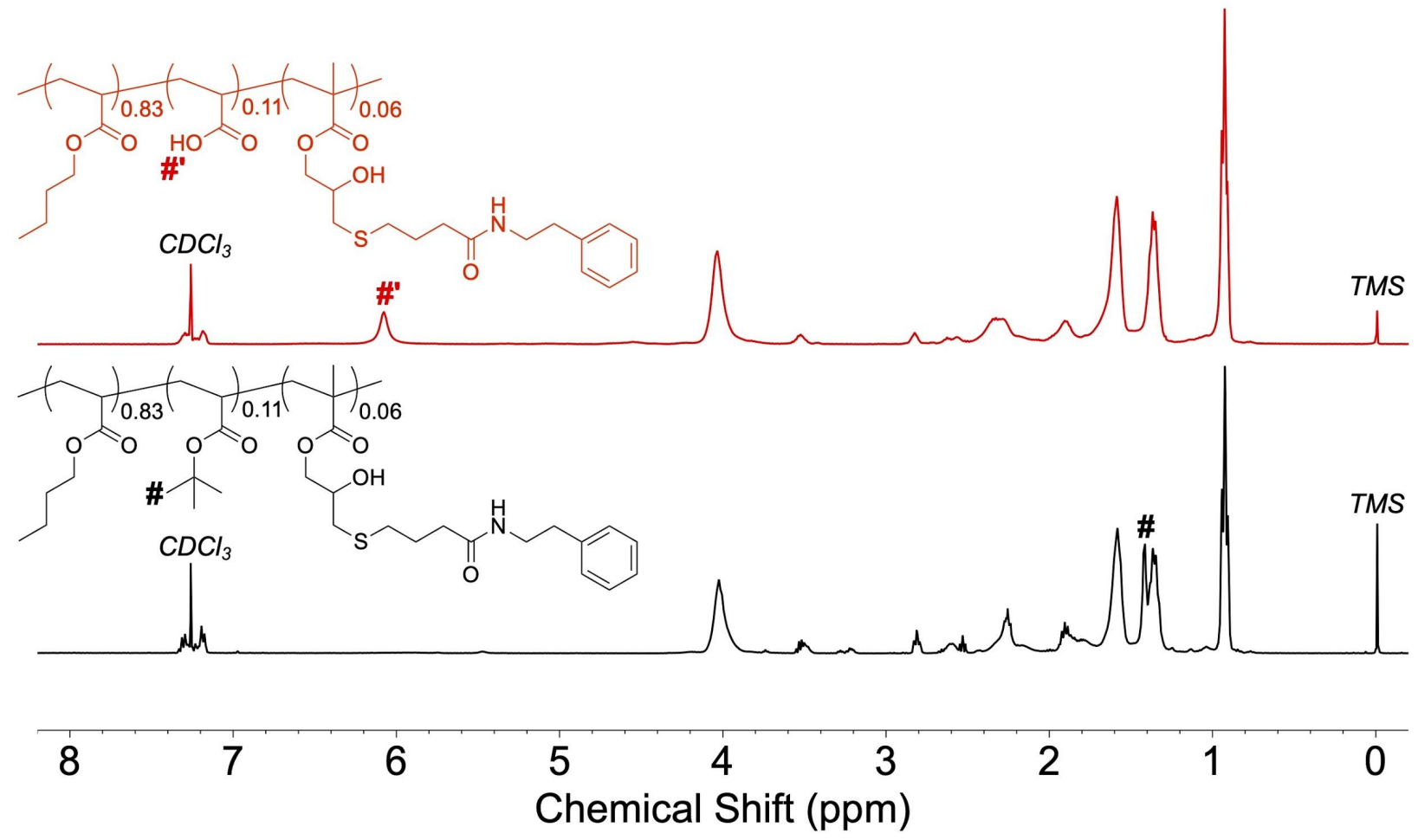

Figure S14. ${ }^{~} \mathrm{H}$ NMR spectrum of benzoic PSAs before (P6, black) and after (P7, red) deprotection process. The pound sign (\#) indicates the peak from the tert-butyl group, which is substituted for the carboxylic group (\#') after deprotection. 


\subsection{Glass Transition Temperature}

Differential scanning calorimetry (DSC) thermograms of each PSA were collected by using Mettler Toledo DSC 1 system (Columbus, OH, US) where heating and cooling rates were maintained to $10{ }^{\circ} \mathrm{C} / \mathrm{min}$ under nitrogen. STARe basic software provided by the vendor was used to computationally calculate the glass transition temperature of the polymer; the second heating curve was employed for the calculation.

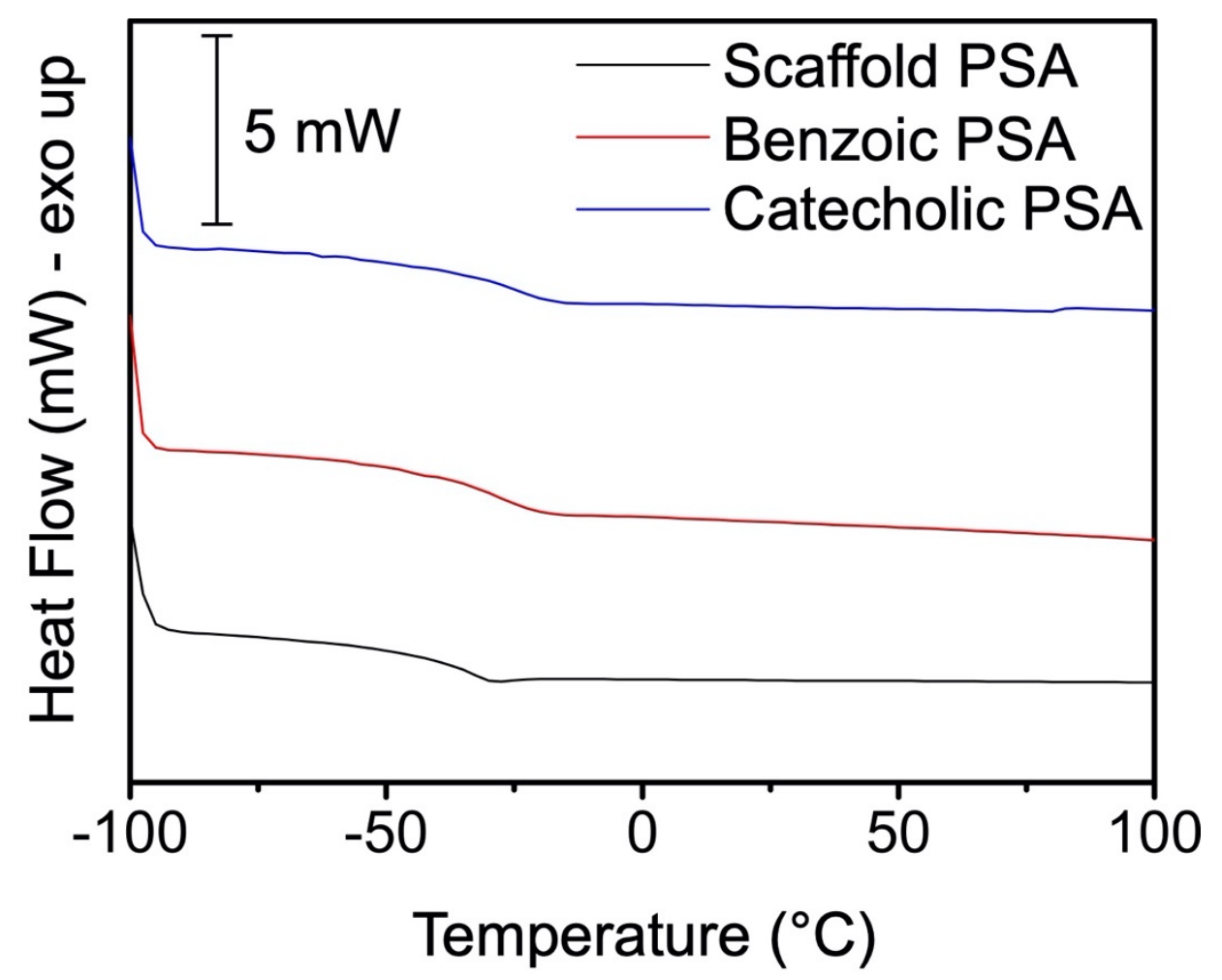

Figure S15. DSC thermograms of scaffold (P1), catecholic (P5) and benzoic (P7) PSAs. The curves were from the second heating cycle.

\section{Mechanical Tests}

Preparation of test specimens: The samples for mechanical tests were prepared by referring to the recent paper from Tiu et al (see the supporting figure 'Figure S8' in the reference paper).(1) In detail, the synthesized PSAs were dissolved in Ar-bubbled methyl ethyl ketone (MEK; Fisher Scientific, Waltham, MA, US, $\geq 99 \%)$ in a $1: 4(\mathrm{w} / \mathrm{v})$ ratio and coated onto $36 \mu \mathrm{m}$ thick poly(ethylene terephthalate) films (Hostaphan RN36, Hoechst, Frankfurt, Germany) using a round-wire K-bar hand coater (Testing Machines, Inc., New Castle, DE) producing $150 \mu \mathrm{m}$ of wet-coating thickness. The coated films were heated inside a $100^{\circ} \mathrm{C}$ oven for 5 minutes to evaporate the solvent and then stored in ambient conditions for 24 hours. The adhesive layer of the coated films was protected by adhering onto a silicone release layer, and further cut into 1-inch strips. Then, the adhesive side of 1-inch strips adhered onto cleaned stainless steel substrates: 
dimensions of adhesive attached to the substrate are ' $25 \mathrm{~mm}(1 \mathrm{inch}) \times 175 \mathrm{~mm}$ ' and '25 mm $\times 25$ $\mathrm{mm}$ ' for peel and shear tests, respectively. Subsequently, a FINAT hand-roller (2 $\mathrm{kg}$, ChemInstruments, Fairfield, OH, US) was rolled over the substrate-adhered tape twice in each direction at a constant speed to establish standard contact between the adhesive and the substrate. As prepared test specimens were stored in ambient conditions for $24 \mathrm{~h}$ prior to the following tests.

$180^{\circ}$ peel and static shear tests: PSA performance was tested based on established FINAT test methods no. 1 (peel test) and 8 (shear test).(2) For the $180^{\circ}$ peel test, the end of a strip was used pulled back at $180^{\circ}$ and tightened to the upper-side holder of the Instron 3345 universal testing system (Norwood, MA, US), and pulled at $300 \mathrm{~mm} / \mathrm{min}$ rate. For the static shear test, the end of the strip was looped through a shear test clip (STC-100, ChemInstruments, Fairfield, OH, US) and attached back to itself. The specimen was put into the substrate holder of a shear tester (SS-RT10, ChemInstruments, Fairfield, OH, US). Subsequently, $500 \mathrm{~g}$ of weight was gently hung on the shear test clip; the time of shear resistance was automatically measured when the weight fell.

Additional Discussion: We have also done the mechanical tests using the PSAs tapes adhered on a high-density polyethylene substrate to judge whether the adhesion/cohesion properties of catecholic PSA can also be improved on low surface energy materials; the results were not promising, similar to what was demonstrated in the previous catecholic PSA study.(1)

\section{Time-dependent Adhesion/Cohesion Behaviors in Catecholic PSAs}

\subsection{Mechanical Tests}

The same mechanical tests as described in section 6 were additionally performed after storage for 6 days in ambient conditions and compared to the original mechanical properties.

\subsection{UV-vis Spectroscopy}

Each polymer was dissolved in Ar-bubbled MEK in a 1:4 (w/v) ratio. Then, the solution $(\sim 200 \mu \mathrm{L})$ was drop-casted and gently spread on a 1-by-1-inch quartz substrate (P/N 26013, Ted Pella, Inc., USA) using a K-bar (150 $\mu \mathrm{m}$ of wet-coating thickness). The solvent on the substrate was dried in a $100{ }^{\circ} \mathrm{C}$ oven for 5 minutes. As prepared PSA deposited the quartz substrate was subsequently stored in ambient conditions for either 1 day or 6 days. The UV-vis spectrum of the PSAs was taken by a UV2600 spectrophotometer (Shimadzu Scientific Instruments, Kyoto, Japan) where bare quartz was simultaneously used as a reference substrate (Figure S16). The collected absorbance spectra were normalized using the Origin Pro software (Version 8, OriginLab, Northampton, MA, US). 

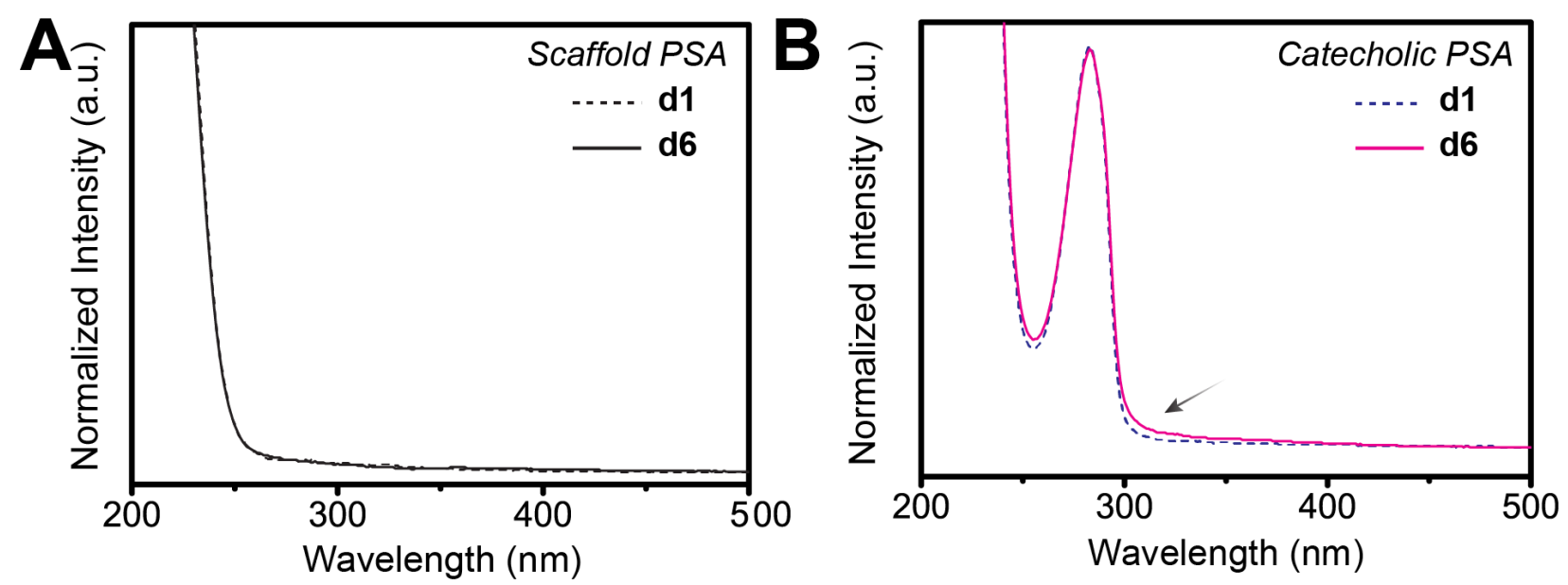

Figure S16. Time-dependent UV-VIS spectrum collected from A) scaffold and B) catecholic PSAs with aging for 1 (d1) or 6 (d6) days.

\section{Statistical Analysis}

P-values were calculated by unpaired two-tailed t-tests by using Microsoft Excel. $\mathrm{p}<0.05$ is generally considered to be statistically significant.

\section{References}

(1) Tiu, B. D. B.; Delparastan, P.; Ney, M. R.; Gerst, M.; Messersmith, P. B. Enhanced Adhesion and Cohesion of Bioinspired Dry/Wet Pressure-Sensitive Adhesives. ACS Appl. Mater. Interfaces 2019, 11 (31), 28296-28306.

(2) FINAT Technical Handbook 6th edn. Test Methods FTM 1, FTM 8 (2001). 